\section{Colonoscopic Detection of Cecal Schistosomiasis Mimicking a Polyp in an Asymptomatic Individual}

Manson's schistosomiasis is a mainly African parasitic infection that affects sixty million people throughout the world. The causative agent is a fluke. Schistosoma mansoni, that infests the venous system (1). Infection takes place during contact with fresh water. The symptoms usually parallel the cycle of the parasite: cutaneous symptoms may occur during the penetration phase, allergic symptoms during the infestation phase, and intestinal symptoms during the mature phase (2). Hepatic and splenic complications are possible, relating to portal hypertension (3). The first two phases can pass unnoticed, but the third is rarely asymptomatic.

We report here the case of a 50 -year-old asymptomatic man in good general condition, with no previous complaints, who underwent screening colonoscopy after his mother contracted cancer of the colon. At endoscopy, no polyps were found, but there was a large ulceration of the ileocecal valve. The ulceration was soft at palpation with the biopsy forceps (Figure 1). The terminal ileum was endoscopically normal, as was the clinical examination. The laboratory values indicated neither inflammatory parameters, nor anemia, or eosinophilia.

Histological examination of the endoscopic biopsies revealed an inflammatory granuloma with giant cells and the remains of a Schistosoma mansoni egg, located centrally (Figure 2). Repeated careful investigation of the patient's history revealed that he had stayed in Central Africa twelve years previously; he had not bathed in fresh water, but had taken showers supplied with water from a lake.

No hepatic, splenic, or portal flow anomaly was evident on Doppler ultrasound. Treatment with praziquantel (one dose of $3000 \mathrm{mg}$ ) was prescribed. A repeated colonoscopy one month later showed complete scar formation of the ileocecal valvule (Figure 3). One year after treatment, the patient was still asymptomatic and well. No further follow-up examinations are planned.

N. Claudel. I. Deshaumes, E. Vincent

Hepatology and Gastroenterology Service, H.I.A. Desgenettes, Lyons, France

\section{References}

1. Iarotski LS, Davis A. The schistosomiasis problem in the world: results of a WHO questionnaire survey. Bull OMS 1981: $59: 115$

2. Warren KS. The pathology, pathobiology and pathogenesis of schistosomiasis. Nature 1978; 273: 609-12.

3. Raia S, Mies S, Naacedo AL. Portal hypertension in schistosomiasis. Clin Gastroenterol 1985: 14: $57-82$.

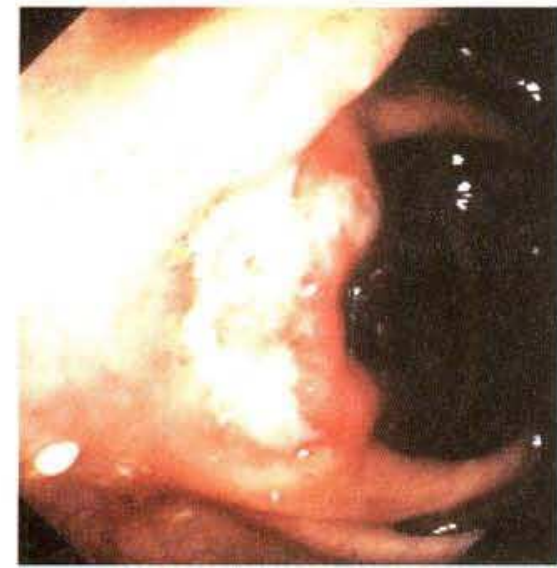

Figure 1: The first colonoscopy. There is ulceration at the ileocecal valvule.

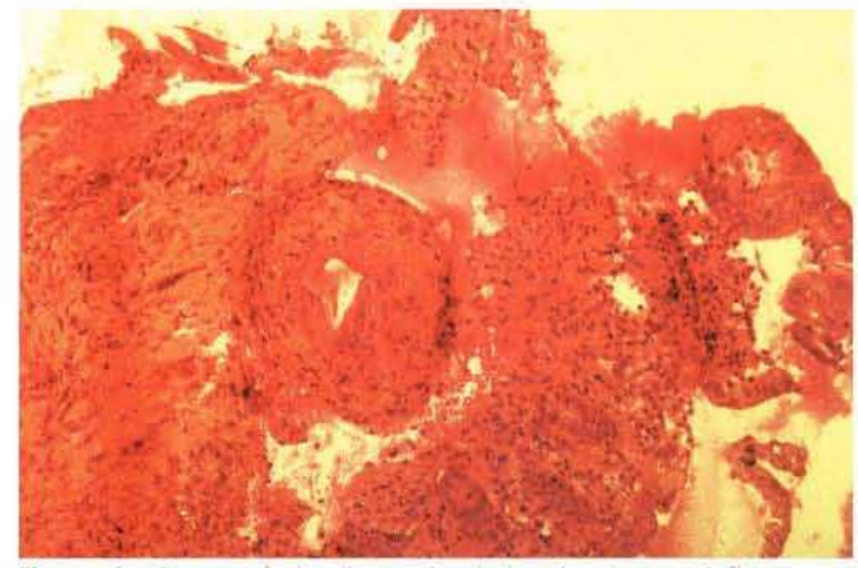

Figure 2: Biopsy of the ileocecal valvule, showing an inflammatory granuloma, with a Schistosoma mansoni ego

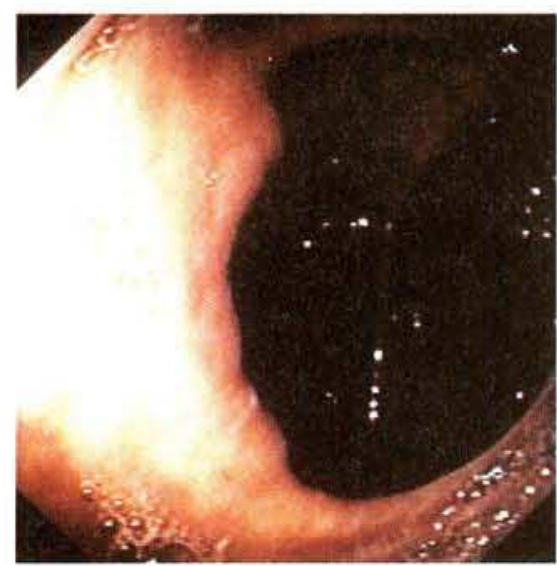

Figure 3: The second colonoscopy. Scar tissue has formed at the ileocecal valvule.

Corresponding Author

N. Claudel. M.D.

Service d'Hépato-Gastro-Entérologie

H.I.A. Desgenettes

108, boulevard Pinel

69275 Lyon

France

Fax: $+33-72366141$ 